\title{
Activation of Cholesterol Synthesis in Preference to Fatty Acid Synthesis in Liver and Adipose Tissue of Transgenic Mice Overproducing Sterol Regulatory Element-binding Protein-2
}

\author{
Jay D. Horton, ${ }^{\star}$ lichiro Shimomura, ${ }^{*}$ Michael S. Brown, ${ }^{*}$ Robert E. Hammer, ${ }^{\ddagger}$ Joseph L. Goldstein, ${ }^{*}$ and Hitoshi Shimano* \\ $*$ Department of Molecular Genetics, ${ }^{\ddagger}$ Department of Biochemistry, and ${ }^{\S}$ Howard Hughes Medical Institute, University of Texas \\ Southwestern Medical Center, Dallas, Texas 75235
}

\begin{abstract}
We produced transgenic mice that express a dominant-positive truncated form of sterol regulatory element-binding protein-2 (SREBP-2) in liver and adipose tissue. The encoded protein lacks the membrane-binding and $\mathrm{COOH}-$ terminal regulatory domains, and it is therefore not susceptible to negative regulation by cholesterol. Livers from the transgenic mice showed increases in mRNAs encoding multiple enzymes of cholesterol biosynthesis, the LDL receptor, and fatty acid biosynthesis. The elevations in mRNA for 3-hydroxy-3-methylglutaryl coenzyme A (HMG CoA) synthase and HMG CoA reductase were especially marked (13-fold and 75-fold, respectively). As a result, the transgenic livers showed a 28-fold increase in the rate of cholesterol synthesis and a lesser fourfold increase in fatty acid synthesis, as measured by intraperitoneal injection of $\left[{ }^{3} \mathrm{H}\right]$ water. These results contrast with previously reported effects of dominant-positive SREBP-1a, which activated fatty acid synthesis more than cholesterol synthesis. In adipose tissue of the SREBP-2 transgenics, the mRNAs for cholesterol biosynthetic enzymes were elevated, but the mRNAs for fatty acid biosynthetic enzymes were not. We conclude that SREBP-2 is a relatively selective activator of cholesterol synthesis, as opposed to fatty acid synthesis, in liver and adipose tissue of mice. (J. Clin. Invest. 1998. 101: 2331-2339.) Key words: cholesterol • low density lipoprotein • sterol regulatory element binding proteins - fatty acids • transgenic mice
\end{abstract}

\section{Introduction}

Cholesterol and fatty acids, the primary lipids synthesized in liver, are subject to different patterns of regulation (reviewed in references 1 and 2). Cholesterol synthesis is high when animals are fed a cholesterol-free diet, and it declines dramatically when cholesterol is fed. Cholesterol synthesis is also enhanced when the demand for cholesterol is increased, as when

Address correspondence to Joseph L. Goldstein, Department of Molecular Genetics, University of Texas Southwestern Medical Center, 5323 Harry Hines Blvd., Room L5-238, Dallas, TX 75235. Phone: 214-648-2141; FAX: 214-648-8804.

Received for publication 2 February 1998 and accepted in revised form 17 March 1998.

J. Clin. Invest.

(C) The American Society for Clinical Investigation, Inc. 0021-9738/98/06/2331/09 \$2.00

Volume 101, Number 11, June 1998, 2331-2339

http://www.jci.org animals consume bile acid-binding resins. In contrast, fatty acid synthesis is driven primarily by the availability of carbohydrate and the stimulating action of insulin. Despite these different patterns of regulation, recent evidence suggests that both biosynthetic pathways can be regulated by a common family of transcription factors designated sterol regulatory element binding proteins (SREBPs) ${ }^{1}$ (reviewed in reference 3 ). SREBPs are members of a broad class of transcription activators that use basic-helix-loop-helix-leucine zipper (bHLH-Zip) motifs to dimerize and to bind DNA. SREBPs are unique in that they are synthesized as membrane-bound precursors that must be liberated by proteolysis in order to enter the nucleus (3). They are also unique in having a tyrosine in place of an otherwise conserved arginine, which allows them to bind to nonpalindromic sterol regulatory elements in preference to classic palindromic E boxes (4).

The membrane-bound precursor forms of SREBPs contain $\sim 1,150$ amino acids. The $\mathrm{NH}_{2}$-terminal segment of $\sim 480$ amino acids includes the bHLH-Zip domain and an acidic transcription activating domain at the extreme $\mathrm{NH}_{2}$ terminus. The bHLH-Zip domain is followed by a membrane attachment domain of $\sim 80$ amino acids that consists of two membrane-spanning segments separated by a short hydrophilic loop. This is followed by a COOH-terminal regulatory domain of $\sim 590$ amino acids. The SREBPs are oriented so that their $\mathrm{NH}_{2}$-terminal and $\mathrm{COOH}$-terminal domains project into the cytoplasm, and the 31-amino acid loop projects into the lumen of the endoplasmic reticulum (3).

The mechanism for regulation of SREBP activity was elucidated through studies of cultured fibroblasts. When such cells are deprived of sterols, a two-step proteolytic process releases the $\mathrm{NH}_{2}$-terminal domains of the SREBPs so that they can enter the nucleus (3). There they bind to sterol regulatory elements in the promoter regions of genes encoding enzymes of cholesterol biosynthesis (HMG CoA reductase, HMG CoA synthase, farnesyl diphosphate synthase, squalene synthase, and others) (5-8). The SREBPs also bind to regulatory sequences in the promoters of at least two genes involved in fatty acid synthesis, acetyl CoA carboxylase and fatty acid synthase $(4,9,10)$, and at least one gene involved in triglyceride synthesis, glycerol-3-phosphate acyltransferase (11). Binding to all these promoters leads to transcriptional activation, which results in increased synthesis of cholesterol and fatty acids. When sterols overaccumulate in cells, the proteolytic release

1. Abbreviations used in this paper: bHLH-Zip, basic-helix-loophelix-leucine zipper; GAPDH, glyceraldehyde-3-phosphate dehydrogenase; HMG CoA, 3-hydroxy-3-methylglutaryl coenzyme A; PEPCK, phosphoenolpyruvate carboxykinase; PPAR- $\gamma$, peroxisomal proliferator-activated receptor; SREBP, sterol regulatory element-binding protein. 
of SREBPs is blocked, the $\mathrm{NH}_{2}$-terminal fragments remain bound to membranes, and transcription of all target genes declines (3).

Three SREBPs are currently recognized (3). Two of these, designated SREBP-1a and SREBP-1c, are produced from a single gene through the use of alternate promoters $(3,12)$. SREBP-1c was cloned independently as a protein that binds to E-boxes and was named ADD-1 (13). SREBP-1a and -1c differ in the length of the first exon, which encodes a portion of the acidic transcription activating domain. This domain is much longer in SREBP-1a than it is in SREBP-1c (12). The other member of the family, designated SREBP-2, has a long transcription activation domain resembling that of SREBP-1a (6). Livers of mice, humans, and hamsters produce relatively large amounts of SREBP-1c and smaller amounts of SREBP-2 and $-1 \mathrm{a}(12,14)$.

The reason for the three isoforms of SREBP is not yet known. The proteins appear to act primarily as homodimers, and heterodimerization is not required for their activity (reference 6 and Pai, J.-T., M.S. Brown, and J.L. Goldstein, unpublished observations). All three SREBPs are capable of activating all of the known target genes, but they do so with different efficiencies. In general, SREBP-1a and SREBP-2 are much more active than SREBP-1c (reference 15 and Pai, J.-T., M.S. Brown, and J.L. Goldstein, unpublished observations).

In an attempt to decipher the roles of individual SREBPs in liver, we have produced transgenic mice that overexpress truncated dominant-positive forms of SREBP-1a (16) or SREBP-1c (15). The encoded proteins terminate at a point between the bHLH-Zip domain and the membrane-attachment domain. Such proteins are never membrane bound, and they enter the nucleus directly without a requirement for proteolysis. As a result, these proteins are not down-regulated when cholesterol accumulates. Transgene expression is driven by the promoter for phosphoenolpyruvate carboxykinase (PEPCK), which is expressed in liver, adipose tissue, and kidney. Expression is induced to high levels in the liver when the mice consume a low carbohydrate/high protein diet $(15,16)$.

Transgenic animals expressing dominant-positive SREBP-1a exhibited a striking phenotype. Their livers were enlarged up to fourfold, owing to the accumulation of massive amounts of triglyceride and cholesteryl ester (16). Despite the buildup of these end-products, the mRNAs encoding multiple enzymes in the cholesterol and fatty acid biosynthetic pathways were markedly elevated. These include the mRNAs for HMG CoA reductase, HMG CoA synthase, farnesyl diphosphate synthase, squalene synthase, acetyl CoA carboxylase, fatty acid synthase, and stearoyl CoA desaturase. The mRNA encoding the LDL receptor was also increased. As a result, the livers of these animals massively overproduced cholesterol and fatty acids as measured by the rate of incorporation of intraperitoneally injected $\left[{ }^{3} \mathrm{H}\right]$ water.

The white adipose tissue of the SREBP-1a transgenic mice was normal in young animals, but it involuted as the animals became older (16). By the age of $4 \mathrm{mo}$, the amount of white adipose tissue had declined by $>90 \%$, as reflected by the amount of epididymal fat.

In contrast to the striking phenotype of the SREBP-1a mice, we observed a much milder phenotype in animals expressing equivalent amounts of the truncated form of SREBP-1c (15). In these animals the liver was not significantly enlarged. The organ showed a modest accumulation of triglyceride and a modest increase in the mRNAs encoding the fatty acid biosynthetic enzymes. The mRNAs encoding the cholesterol biosynthetic enzymes were not elevated.

In the current study we have extended these observations to SREBP-2. We have produced transgenic mice that overexpress a truncated dominant-positive form of SREBP-2 under control of the PEPCK promoter. The pathways of cholesterol biosynthesis and fatty acid biosynthesis are both activated in the SREBP-2 transgenic animals, but the ratio of cholesterol biosynthesis relative to fatty acid biosynthesis is much higher in these animals than in the SREBP-1a transgenics. This is attributable to a marked increase in the mRNAs for HMG CoA synthase and HMG CoA reductase. These data indicate that the major function of SREBP-2 is to activate cholesterol biosynthesis in preference to fatty acid synthesis in liver and adipose tissue.

\section{Methods}

Materials and general methods. Sequencing reactions were performed on an Applied Biosystems 373A DNA Sequencer (Foster City, CA) by the dideoxy chain termination method. The content of cholesterol and triglyceride in plasma and liver was measured as described previously (16). Transgenic mice expressing SREBP-1c436 (encoding amino acids $1-436$ of human SREBP-1c) (15) and SREBP-1a460, line B (encoding amino acids 1-460 of human SREBP-1a) (16) were described in the indicated reference.

Measurement of hepatic unesterified and esterified cholesterol. Total lipids were extracted from 200-250-mg aliquots of liver. Unesterified and esterified cholesterol were separated on 500-mg silica columns (Cat no. 1211-3036; Varian, Harbor City, CA) as described (17) and quantified by gas liquid chromatography (18).

Plasmid construction. The expression plasmid pPEPCK-SREBP2(1-468) contained the rat PEPCK promoter $(2.4 \mathrm{~kb})$ fused to a cDNA encoding amino acids 1-468 of human SREBP-2 (6) followed by two consecutive stop codons. It was constructed as previously described for pPEPCK-SREBP-1a460 except that the cDNA fragment was produced by PCR and ligated into the SmaI site of the pCMVPEPCK promoter (16). The PCR reaction was performed using pSREBP-2 (6) as a template, Pfu polymerase, a 5' primer corresponding to $\mathrm{bp}-74$ to -45 in the $5^{\prime}$ untranslated region of human SREBP-2 (5'-GGGCGGTGGCGACGGCACCGCCCCCGCGTCT-3'), and a $3^{\prime}$ primer corresponding to amino acids $459-468$ followed by two consecutive stop codons (5'-GGGTCATCAGTCTGGCTCATCTTTGACCTTTGCATCATC-3'). The integrity of the construct was confirmed by DNA sequencing.

Production and maintenance of transgenic mice. Techniques used for generating transgenic mice were previously described (16). A total of 1,502 fertilized eggs that were microinjected with the SalI-SphI fragment of pPEPCK-SREBP-2(1-468) survived to the two-cell stage. Among the 184 offspring, $23 \%$ had integrated the transgene as determined by dot blot hybridization of DNA from tail homogenates. Of 42 DNA-positive mice subjected to partial hepatectomy, $30 \%$ produced truncated SREBP-2 as determined by immunoblot analysis. Mice with high levels of transgene expression in liver were bred to C57BL/6J $\times$ SJL F1 mice, and two independent lines of SREBP2(1-468) mice were established, designated lines A and B. To obtain higher expression, hemizygotes of line B were mated to produce homozygotes for the transgene. Hemizygous mice from line A and homozygous mice from line B were used for all studies. Mice were housed in colony cages and maintained on a 14-h light/10-h dark cycle. Transgenic mice and nontransgenic littermates were fed a low carbohydrate/high protein diet (No. 5789C-3) from Purina Mills Inc. (St. Louis, MO) containing $71 \%$ (wt/wt) casein and $4.25 \%$ sucrose for $2 \mathrm{wk}$ to induce maximal expression of the transgene (19). All mice were fasted $4 \mathrm{~h}$ before death during the early phase of the light cycle. 
Immunoblotting. Nuclear extracts were prepared from pooled liver samples as previously described (16). Each sample (30 $\mu \mathrm{g}$ protein) was subjected to $8 \%$ SDS-PAGE, electrophoretically transferred to Hybond $\mathrm{C}$ extra membranes (Amersham Corp., Arlington Heights, IL), and incubated with $5 \mu \mathrm{g} / \mathrm{ml}$ of rabbit anti-human SREBP-2 IgG, which was raised against amino acids 48-403 of human SREBP-2 (6). Immunoblot analysis was carried out with the Enhanced Chemiluminescence (ECL) Western Blotting Detection System Kit (Amersham Corp.) with a horseradish peroxidase-labeled donkey anti-rabbit IgG antibody (Amersham Corp.) as previously described (16).

Blot hybridization of RNA. Total RNA was prepared from mouse liver and epididymal fat pad using the RNeasy ${ }^{\mathrm{TM}}$ Total RNA kit (Qiagen Inc., Chatsworth, CA) and the acid guanidinium thiocyanate-phenol-chloroform method (20), respectively. Equal aliquots of total RNA from each group of mice were pooled (total, $15 \mu \mathrm{g}$ ) and subjected to Northern blot analysis with the indicated cDNA probes prepared as previously described $(15,16,21)$. A HindIII fragment $(1.3 \mathrm{~kb})$ of the human SREBP-2 cDNA was used as a cDNA probe for the transgene. A probe for mouse PPAR- $\gamma(22)$ was prepared by reverse transcriptase-PCR using mouse liver poly(A) ${ }^{+}$RNA as template as previously described for other probes (16). The $5^{\prime}$ and $3^{\prime}$ primers were 5'-ACAAGACTACCCTTTACTGAAATTACCATG-3' and 5'-GGCACTTCTGAAACCGACAGTACTGACATTTATTT-3', respectively. The bands on the Northern blots were quantified by exposure of the filter to a BAS1000 Fuji PhosphorImager (Tokyo, Japan), and the results were normalized to the signal generated from GAPDH mRNA.

RNase protection assay. cRNA probes were prepared from cDNA fragments for human SREBP-1 and SREBP-2 that were produced by PCR amplification using the plasmids pPEPCK-SREBP1a460 (16) and pPEPCK-SREBP-2(1-468) (this paper) as templates, respectively. The following primers were used. Human SREBP-1: 5' primer, 5'-GGCACAGACCCTGCCAGCC-3'; and 3' primer, 5'-CTGGGGTAGCCTAACACAGG-3' (5). Human SREBP-2: 5' primer, $5^{\prime}$-GTAGCAGCAGCGGCAGCAGTGG-3'; and $3^{\prime}$ primer, 5'-AAAGGCTGCTGGATGATCCTCG-3' (6). The cDNA fragment for human SREBP-1 is identical in DNA sequence for isoforms SREBP-1a and -1c. HindIII and EcoRI sites were added to both 5' and $3^{\prime}$ primers, respectively. The RNase protection assay was done as previously described $(12,14)$. For comparison of transgene expression levels in livers of SREBP-1a, -1c, and -2 transgenic mice, each probe was designed so that each protected fragment would contain the same number of ${ }^{32} \mathrm{P}$ atoms (i.e., 69).

Cholesterol and fatty acid synthesis in vivo. Five 12-wk-old mice homozygous for the human SREBP-2 transgene (line B) and wildtype mice from the same litters (three females and one male for each group) were fasted for $4 \mathrm{~h}$ and killed during the early light cycle. The rates of incorporation of intraperitoneally injected $\left[{ }^{3} \mathrm{H}\right]$ water into cholesterol and fatty acid were measured in liver as previously described (16)

\section{Results}

To produce transgenic mice expressing a dominant-positive form of SREBP-2, we constructed a plasmid encoding amino acids 1-468 of human SREBP-2 under control of the PEPCK promoter. The encoded protein includes the bHLH-Zip DNA binding domain and the $\mathrm{NH}_{2}$-terminal acidic transactivation domain, but it lacks the membrane-attachment domain and the $\mathrm{COOH}$-terminal regulatory domain. Previous studies in tissue culture demonstrated that such truncated SREBPs enter
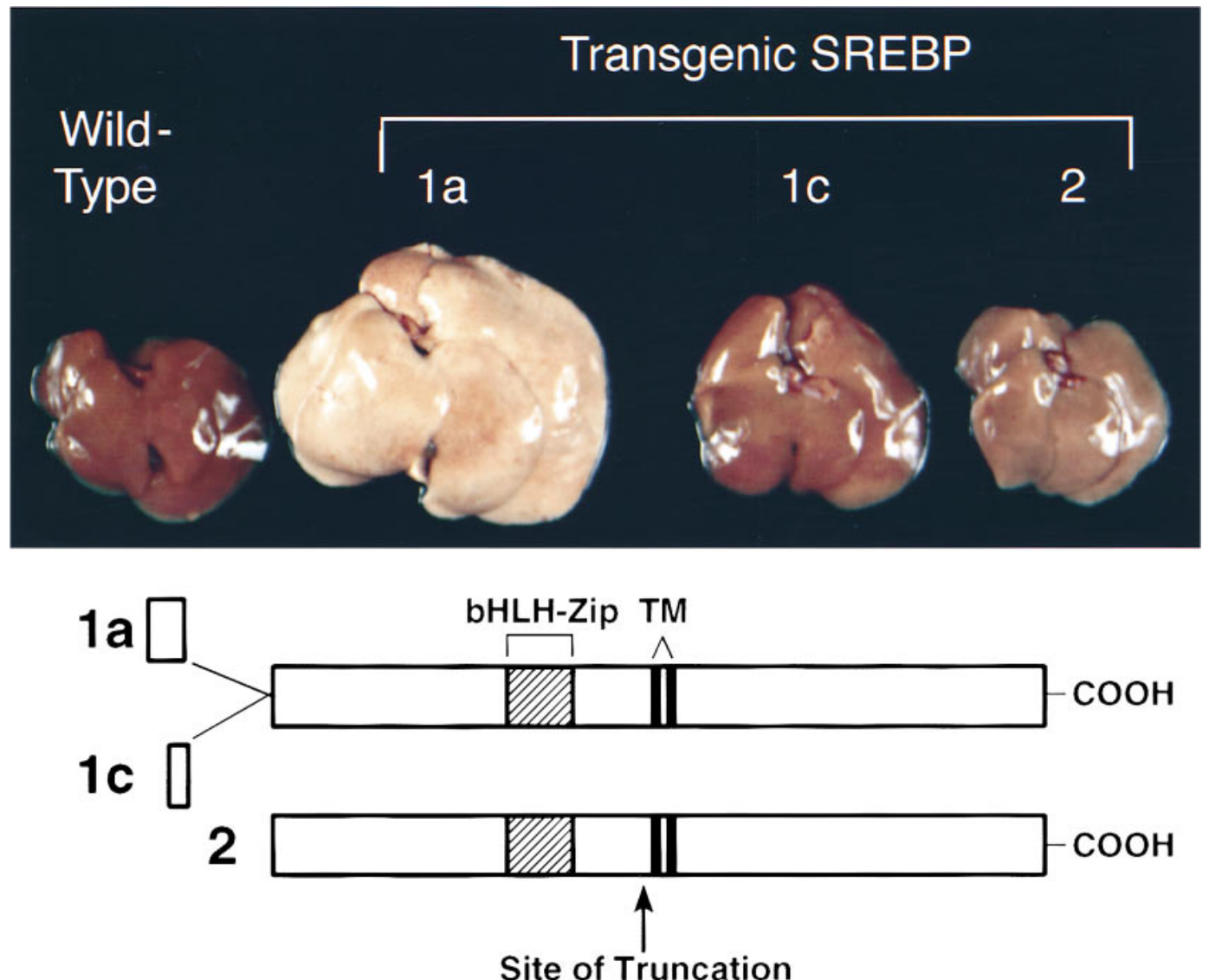

Figure 1. Photograph of livers from 12 -wk-old wild-type mice and transgenic mice expressing truncated dominant-positive SREBP-1a, -1c, and -2. The SREBP-1a (line $B)$ and SREBP-1c mice were described in references 15 and 16 . The SREBP-2 mouse was from line B. All animals were fed the low carbohydrate/high protein diet for $2 \mathrm{wk}$ before killing. (Lower panel) schematic diagram of the domain structure of SREBP-1a, $-1 \mathrm{c}$, and -2 , illustrating the site of truncation used for construction of the transgenes. TM, transmembrane segments. 


\begin{tabular}{lccc}
\hline & & & TgSREBP-2 mice \\
\cline { 2 - 3 } \multicolumn{1}{c}{ Parameter } & Wild-type mice & Line A & Line B \\
\hline Number and sex & Five males & Five males & Five males \\
Body weight (grams) & $27.3 \pm 0.5$ & $27.1 \pm 1.0$ & $24.9 \pm 1.1$ \\
Liver weight (grams) & $1.5 \pm 0.11$ & $1.5 \pm 0.03$ & $1.5 \pm 0.09$ \\
Liver weight/body weight & $0.053 \pm 0.003$ & $0.056 \pm 0.002$ & $0.058 \pm 0.002$ \\
Epididymal fat pad weight (grams) & $0.24 \pm 0.04$ & $0.40 \pm 0.06$ & $0.27 \pm 0.02$ \\
Epididymal fat weight/body weight & $0.009 \pm 0.001$ & $0.015 \pm 0.002$ & $0.011 \pm 0.001$ \\
Liver cholesterol content (mg/gram) & $2.2 \pm 0.09$ & $3.6 \pm 0.17(P<0.001)^{*}$ & $5.3 \pm 0.57(P<0.01)^{*}$ \\
Liver triglyceride content (mg/gram) & $7.2 \pm 1.4$ & $10.7 \pm 2.7$ & $28.3 \pm 6.9(P<0.05)^{*}$ \\
Total plasma cholesterol (mg/dl) & $87 \pm 6.4$ & $111 \pm 4.0(P<0.05)^{*}$ & $70 \pm 8.5(P<0.05)^{*}$ \\
Total plasma triglycerides (mg/dl) & $119 \pm 17$ & $101 \pm 12$ & $63 \pm 5.5$ \\
& &
\end{tabular}

Each value represents the mean \pm SEM of five 16-wk-old male mice. Wild-type and line A hemizygous transgenic mice were obtained from the same two litters. Line B transgenic mice were homozygous for the transgene and obtained from four littermates from hemizygote matings. All mice were fed the low carbohydrate/high protein diet for $2 \mathrm{wk}$ and fasted $4 \mathrm{~h}$ before death. *Values in parentheses denote the level of statistical significance (Student's $t$ test) between wild-type and the indicated line of transgenic mice. For parameters in which the level of significance is $P>0.05$, no values are shown.

the nucleus directly without a requirement for proteolysis (23). The site of truncation in SREBP-2 is equivalent to the sites of truncation in the SREBP-1a and -1c proteins that have been studied previously in transgenic animals (Fig. 1, lower panel).

The SREBP-2 transgene construct was injected into mouse eggs, and we derived two independent lines of transgenic mice, designated A and B. To increase expression, mice from line B were bred to homozygosity for the transgene. The line A mice were maintained in the hemizygous state. Fig. 1 (upper panel) compares the appearance of the liver of one representative mouse from SREBP-2, line B and livers from previously described transgenic mice expressing dominant-positive forms of SREBP-1a and -1c $(15,16)$. As reported previously, the livers from the SREBP-1a animals were grossly enlarged and pale, owing to massive accumulation of cholesteryl esters and triglycerides (16), whereas the SREBP-1c livers were generally indistinguishable from wild-type (15). Livers from the SREBP-2 animals were not visibly enlarged, but they exhibited a distinct pale color that was consistently different from that of wild-type mice (Fig. 1, upper panel).

Table I compares various phenotypic parameters in wildtype mice and SREBP-2 transgenic mice of lines A and B. Statistically significant changes were confined to the liver and plasma. Livers from the SREBP-2 transgenic animals were normal in weight, but they contained increased amounts of cholesterol and triglyceride. The levels of both lipids were higher in line B than in line A. The plasma cholesterol level was increased slightly in the line A animals and decreased slightly in the line B animals, a finding of questionable biologic significance. Plasma triglyceride levels were reduced in the transgenic mice, but this was not statistically significant, owing to the large variation in triglyceride levels in the wild-type mice. Importantly, the weight of adipose tissue, as reflected in the epididymal fat pad, was normal in the SREBP-2 transgenic mice, a finding that contrasts with the marked decline observed in the SREBP-1a transgenics (16).

Livers from line B mice expressed a somewhat higher level of truncated SREBP-2 protein as compared with livers from the line A animals (1.3-fold) as determined by immunoblotting (Fig. 2). Similar results were seen in one other experiment in which these levels were compared.

In the Northern blotting experiment of Fig. 3, the livers of the line $\mathrm{B}$ mice contained about threefold more transgene SREBP-2 mRNA than the livers of the line A mice (Fig. 3, upper left panel). A similar trend was seen when we examined levels of mRNA for putative SREBP target genes. Elevations were observed in the levels of mRNA for endogenous SREBP-1 and -2 , the LDL receptor, and multiple genes of cholesterol biosynthesis, including HMG CoA synthase, HMG CoA reductase, farnesyl diphosphate (FPP) synthase, and squalene synthase. The magnitude of the increase in $\mathrm{HMG} \mathrm{CoA}$ reductase mRNA is particularly noteworthy (16-fold in line A and 75-fold in line B). Elevated levels of mRNAs for fatty acid biosynthetic enzymes were also observed, including acetyl CoA carboxylase, fatty acid synthase, and stearoyl CoA desaturase. In each case the elevation was greater for the line $\mathrm{B}$ animals as compared with the line A animals. We also observed an elevation in the mRNA encoding the microsomal triglyceride transfer protein (MTP), which participates in the synthesis of tri-

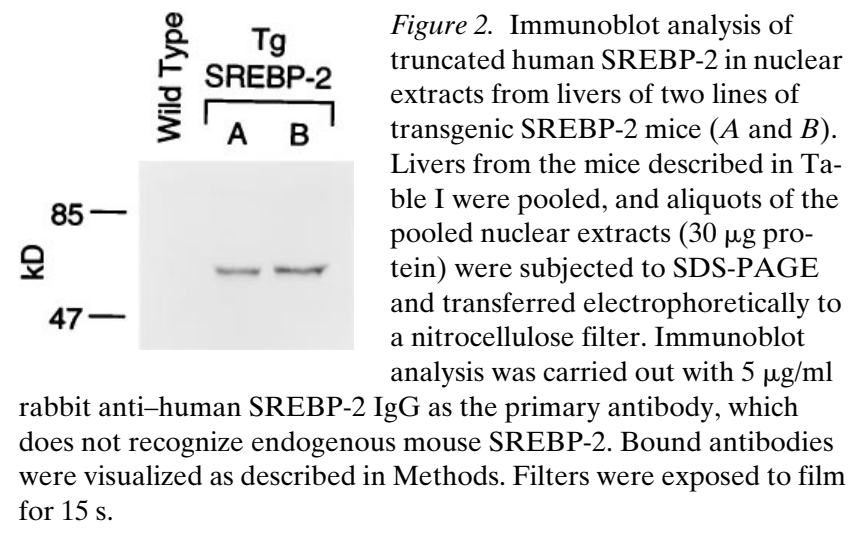

Figure 2. Immunoblot analysis of SREBP-2 in nuclea tivers of two lines of ble I were pooled, and aliquots of the pooled nuclear extracts $(30 \mu \mathrm{g}$ protein) were subjected to SDS-PAGE and transferred electrophoretically to a nitrocellulose filter. Immunoblot analysis was carried out with $5 \mu \mathrm{g} / \mathrm{m}$ does not recognize endogenous mouse SREBP-2. Bound antibodies for $15 \mathrm{~s}$. 


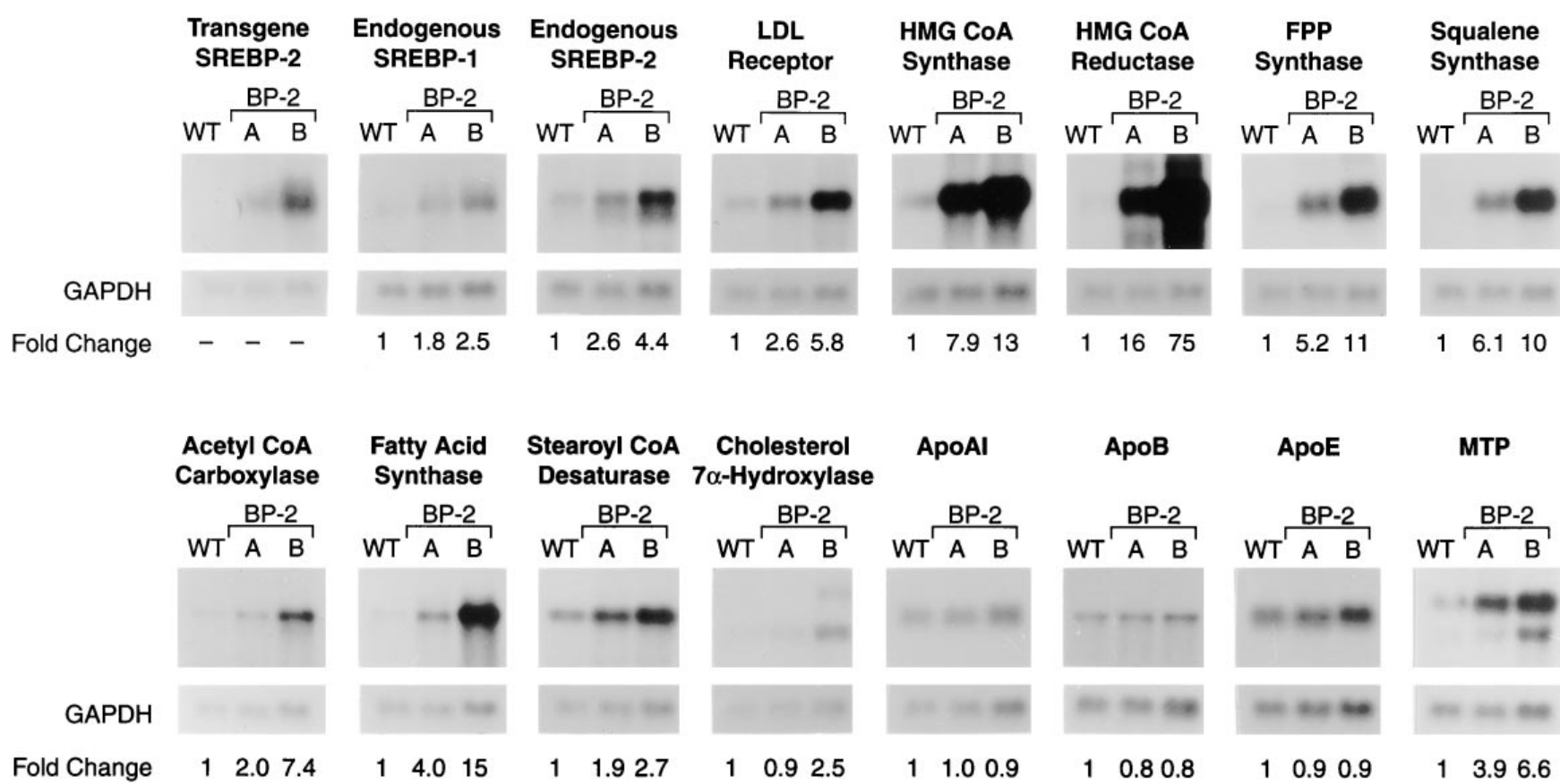

Figure 3. Amounts of various mRNAs in livers of wild-type (WT) mice and two lines of transgenic SREBP-2 mice $(A$ and $B)$ as measured by blot hybridization. Total liver RNA isolated from mice described in Table I was pooled (five mice per group), and $15-\mu \mathrm{g}$ aliquots were subjected to electrophoresis and blot hybridization with the indicated ${ }^{32} \mathrm{P}$-labeled probe and with a control ${ }^{32} \mathrm{P}$-labeled probe directed against GAPDH. The amount of radioactivity in each band was quantified as described in Methods. The fold increase in each mRNA of transgenic mice, relative to that of wild-type control mice, was calculated after correction for loading differences with GAPDH. These values are shown below each blot. The probe for stearoyl CoA desaturase (SCD) was the mouse SCD-1 cDNA fragment that can detect both SCD-1 and SCD-2 mRNAs (16). FPP, farnesyl diphosphate; MTP, microsomal triglyceride transfer protein.

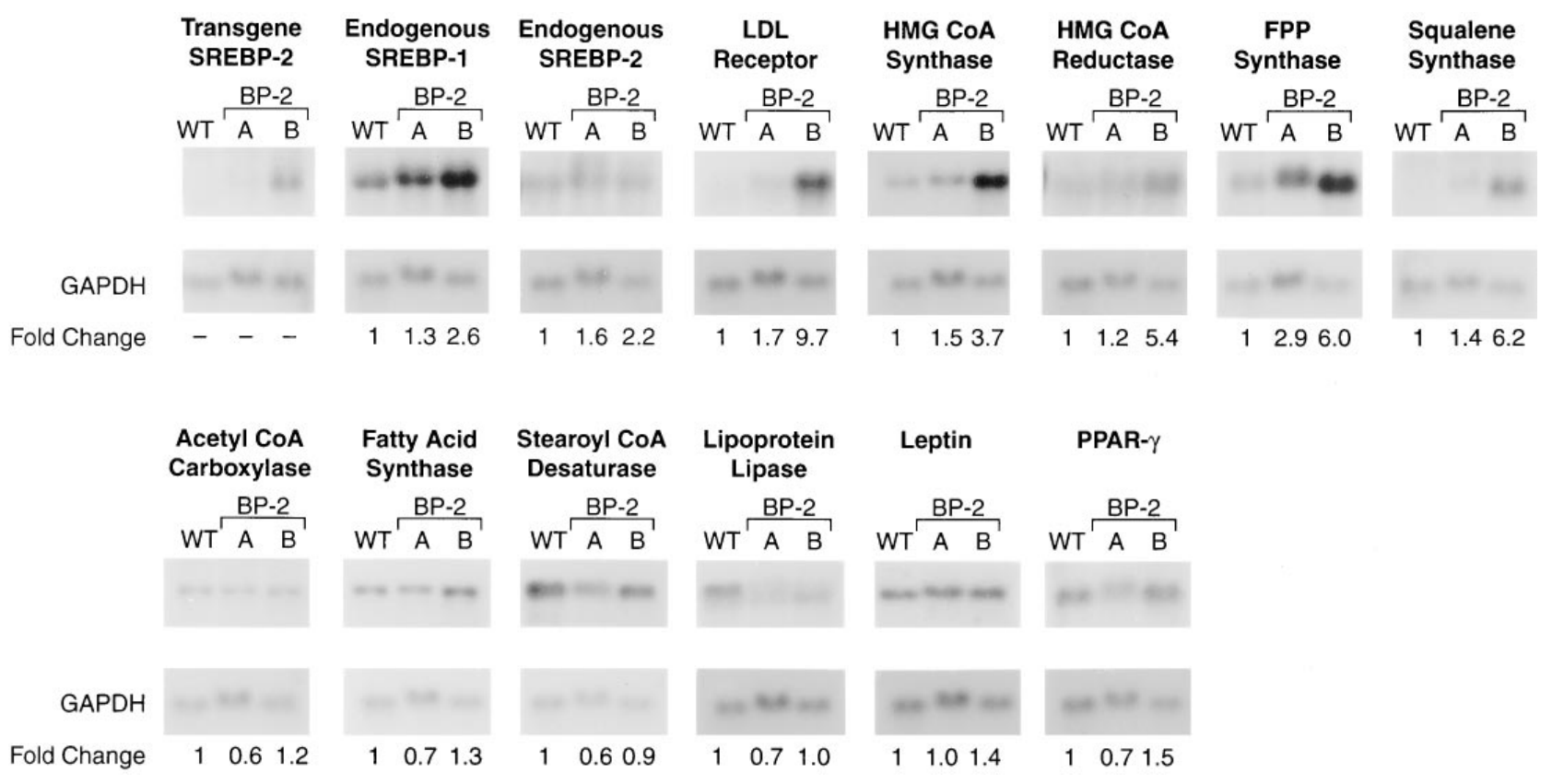

Figure 4. Amount of various mRNAs in white adipose tissues of wild-type (WT) and two lines of transgenic SREBP-2 mice $(A$ and $B)$ as measured by blot hybridization. Total RNA isolated from epididymal fat pads of mice described in Table I was pooled, and 15- $\mu \mathrm{g}$ aliquots were subjected to electrophoresis and blot hybridization with the indicated ${ }^{32} \mathrm{P}$-labeled probe and with a control ${ }^{32} \mathrm{P}$-labeled probe directed against GAPDH. The amount of radioactivity in each band was quantified as described in Methods. The fold increase in each mRNA of transgenic mice, relative to that of wild-type control mice, was calculated after correction for loading differences with GAPDH. These values are shown below each blot. The probe for stearoyl CoA desaturase (SCD) was the mouse SCD-1 cDNA fragment that can detect both SCD-1 and SCD-2 mRNAs (16). FPP, farnesyl diphosphate. 


\begin{tabular}{|c|c|c|c|c|c|}
\hline \multirow{3}{*}{ Genotype of mice } & \multirow{3}{*}{$\begin{array}{c}\text { Liver weight } \\
\text { grams }\end{array}$} & \multicolumn{4}{|c|}{ Incorporation of $\left[{ }^{3} \mathrm{H}\right]$ water into } \\
\hline & & \multicolumn{2}{|c|}{ Digitonin-precipitable sterols } & \multicolumn{2}{|c|}{ Fatty acids } \\
\hline & & $\mu \mathrm{mol} / \mathrm{h}$ per gram & $\mu m o l / h$ per organ & $\mu m o l / h$ per gram & $\mu m o l / h$ per organ \\
\hline Wild-type $(n=5)$ & $1.04 \pm 0.04$ & $0.35 \pm 0.1$ & $0.36 \pm 0.1$ & $4.0 \pm 0.4$ & $4.1 \pm 0.5$ \\
\hline TgSREBP-2 $(n=5)$ & $1.37 \pm 0.07$ & $9.9 \pm 1.2$ & $13.4 \pm 1.2$ & $15.3 \pm 2.9$ & $21.7 \pm 5.1$ \\
\hline
\end{tabular}

Each value represents the mean \pm SEM of four female and one male 12 -wk-old littermate mice of the indicated genotype. The transgenic SREBP-2 mice were from line B. All mice were fed the low carbohydrate/high protein diet for $2 \mathrm{wk}$ and were fasted $4 \mathrm{~h}$ before the experiment. $[\mathrm{H}]$ water was injected intraperitoneally, and $1 \mathrm{~h}$ later the liver was removed for measurement of its content of ${ }^{3} \mathrm{H}$-labeled digitonin-precipitable sterols and fatty acids as described in Methods. All differences between wild-type and transgenic SREBP-2 mice were statistically significant by the Student's $t$ test $(P<0.02)$.

glyceride-rich VLDL (24). Inasmuch as this gene has not been studied previously as a target for SREBPs, we do not know whether the elevation is a direct effect of SREBP-2 action or whether it is secondary to the increased lipid content of the liver. The mRNA for cholesterol 7- $\alpha$ hydroxylase was elevated slightly in the line B animals. In agreement with previous observations with the other transgenic SREBP mice $(15,16)$, we found no elevation in the levels of mRNA for apoproteins of the plasma lipoprotein transport system (namely, apo AI, apo $\mathrm{B}$, and apo E) in livers of the mice expressing SREBP-2.

The PEPCK promoter that drives expression of the SREBP-2 transgene is active in adipose tissue as well as in liver. In the experiment of Fig. 4, we compared the levels of various mRNAs in epididymal fat pads of wild-type mice and transgenic lines $\mathrm{A}$ and $\mathrm{B}$. The amount of transgene expression was higher in the line B animals than in the line A animals, and this was reflected in elevated levels of mRNA for the LDL receptor and the cholesterol biosynthetic enzymes. Surprisingly, we did not observe consistent elevations in mRNAs for acetyl CoA carboxylase, fatty acid synthase, or stearoyl CoA desaturase in adipose tissue. We also did not observe significant effects on mRNAs for relatively fat-specific genes such as lipoprotein lipase, leptin, or PPAR- $\gamma$. Thus, in adipose tissue the truncated SREBP-2 has a relatively specific stimulatory effect on transcription of genes involved in cholesterol supply as opposed to fatty acid input.

In livers of the SREBP-2 transgenic mice, the increases in mRNAs encoding lipogenic enzymes were reflected in elevated rates of lipid synthesis (Table II). To make these measurements, wild-type and SREBP-2 transgenic mice (line B) were injected intraperitoneally with $\left[{ }^{3} \mathrm{H}\right]$ water, and the incorporation into digitonin-precipitable sterols and fatty acids was measured in liver extracts $1 \mathrm{~h}$ later. These studies revealed a massive increase in sterol synthesis (28-fold) and a lesser increase in fatty acid synthesis (fourfold) in the SREBP-2 transgenics when expressed on the basis of liver weight. The increase in cholesterol synthesis was associated with an 11-fold increase in cholesteryl ester content in the SREBP-2 transgenic livers (Fig. 5).

\section{Discussion}

The current experiments complete a series in which dominantpositive truncated versions of all three SREBPs were expressed in liver and adipose tissue of transgenic mice under control of the PEPCK promoter. Figs. 6-9 compare the results with respect to the liver. The experiments in each strain were performed at different times, but the animals were all approximately the same age, and they were all fed the low carbohydrate/high protein diet for $2 \mathrm{wk}$ to induce transgene expression before killing. Fig. 6 compares the levels of transgene expression as determined by RNase protection assays using probes that were adjusted to contain the same number of ${ }^{32} \mathrm{P}$ atoms in each protected fragment. The level of transgene-encoded mRNA was higher for SREBP-1a than for SREBP-1c or SREBP-2. Although these differences preclude a direct comparison of each isoform's absolute effect on the absolute rate of lipid synthesis, they do allow a valid comparison of the relative effect of each isoform on cholesterol synthesis versus triglyceride synthesis.

Although all three SREBP isoforms tended to stimulate expression of multiple genes involved in lipid synthesis, the relative degree of stimulation varied in ways that indicated differential effects on the pathways of cholesterol as opposed to fatty acid synthesis. As shown in Fig. 7, SREBP-2 was more effective than SREBP-1a in increasing the amounts of the three

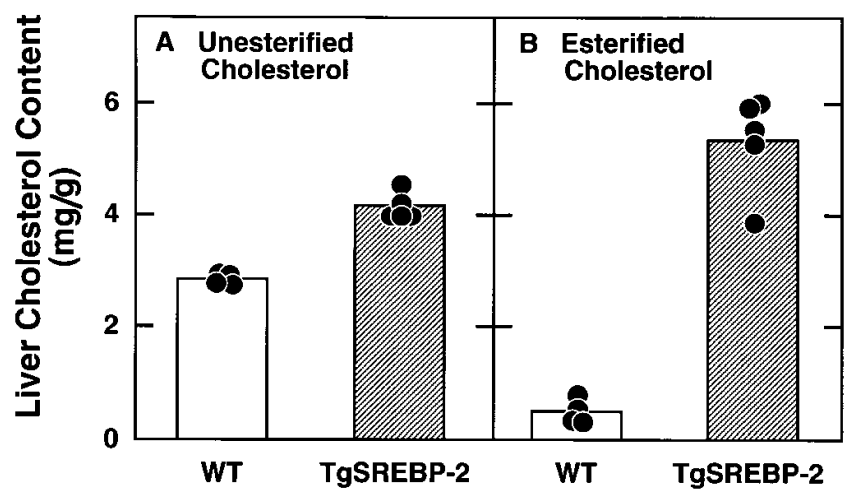

Figure 5. Content of unesterified $(A)$ and esterified $(B)$ cholesterol in livers of wild-type and transgenic SREBP-2, line B mice. Four 16-wkold wild-type female mice and five transgenic SREBP-2, line B littermate female mice were fed the low carbohydrate/high protein diet for $2 \mathrm{wk}$ and fasted $4 \mathrm{~h}$ before study. Aliquots of liver (200-250 mg) were taken for measurement of unesterified and esterified cholesterol as described in Methods. Each circle denotes the value from an individual animal, and the bar represents the mean. 


\begin{tabular}{|c|c|c|c|}
\hline \multirow{2}{*}{$\begin{array}{c}\text { Source of } \\
\text { Liver }\end{array}$} & \multicolumn{3}{|c|}{ Transgenic } \\
& \multicolumn{2}{|c|}{ SREBP- } \\
\cline { 2 - 4 } & 1c & 1a & 2 \\
\hline Lane & A & B & C \\
\hline
\end{tabular}

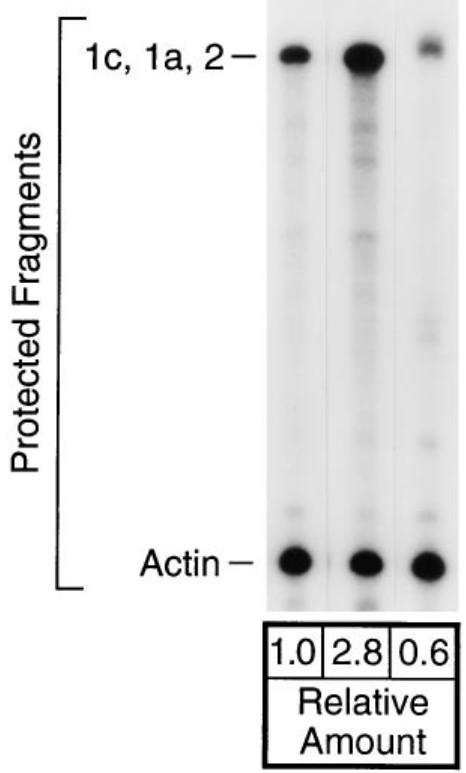

Figure 6. Relative amounts of mRNA encoding truncated SREBP-1c (lane $A$ ), SREBP-1a (lane $B$ ), and SREBP-2 (lane $C$ ) in livers from transgenic mice as measured by RNase protection. Total RNA from the livers of mice expressing the truncated transgenes encoding SREBP-1c (15), SREBP-1a (16), and SREBP-2, line B (this paper) was isolated, and equal aliquots $(10 \mu \mathrm{g})$ were hybridized at $68^{\circ} \mathrm{C}$ to the appropriate ${ }^{32} \mathrm{P}$-labeled cRNA probe as described in Methods. Protected fragments were separated by gel electrophoresis and exposed to film for $8 \mathrm{~h}$ at $-80^{\circ} \mathrm{C}$ with an intensifying screen. The radioactivity in the gels was quantified as described in Methods, normalized to the $\beta$-actin signal, and expressed as the fold change relative to the value in SREBP-1c mice, which was arbitrarily set at 1.0 .

mRNAs of the cholesterol biosynthetic pathway (HMG CoA synthase, HMG CoA reductase, and squalene synthase), whereas SREBP-1a was more effective than SREBP-2 with respect to the two mRNAs of fatty acid biosynthesis (acetyl CoA carboxylase and fatty acid synthase). Both SREBPs were approximately equipotent with respect to the LDL receptor. SREBP-1c was much less effective than either SREBP-1a or SREBP-2 in stimulating transcription of all of the genes.

The differential effects of the three SREBPs on mRNA transcription were reflected in differential rates of lipid synthe-

sis from $\left[{ }^{3} \mathrm{H}\right]$ water (Fig. 8). SREBP-2 stimulated sterol synthesis more profoundly than SREBP-1a (Fig. $8 A$ ), and the reverse was observed with respect to fatty acid synthesis (Fig. 8 $B)$. As shown in Fig. 9, SREBP-1a raised the hepatic content of triglycerides more than it did cholesterol, and therefore it raised the triglyceride/cholesterol ratio (Fig. $9 C$ ). On the other hand, SREBP-2 raised the content of both lipids to approximately the same extent, and therefore the triglyceride/cholesterol ratio remained about the same as in the control animals (Fig. 9 C). Interestingly, although SREBP-1c was much less active than SREBP-1a or SREBP-2, it raised the triglyceride content of the livers while leaving the cholesterol content unchanged. Thus SREBP-1c, like SREBP-1a, increased the triglyceride/cholesterol ratio.

The massive increase in cholesterol synthesis in the SREBP-2 transgenic mice (28-fold) correlates with the massive increase in the amount of mRNA for HMG CoA reductase (75-fold in the line $\mathrm{B}$ mice). This was coupled with an 11-fold increase in the amount of mRNA for HMG CoA synthase, which produces the substrate for the HMG CoA reductase reaction. These results emphasize the importance of these two enzymes as rate-controlling steps for the overall pathway of cholesterol biosynthesis.

Considered together, the comparative results of Figs. 7-9 indicate that overexpression of dominant positive forms of SREBP-1a and SREBP-1c favors triglyceride synthesis as opposed to cholesterol synthesis, whereas expression of SREBP-2 tends to favor the synthesis of cholesterol over triglyceride in livers of transgenic mice.

The experiments with dominant positive transgenes are conducted under highly artificial experimental conditions, yet they have implications for normal physiology. The results suggest that SREBP-1a, -1c, and -2 may have subtly distinct roles in the liver. Under normal conditions the mouse liver produces relatively large amounts of SREBP-1c relative to SREBP-1a (12). The same is true of the human (12) and the hamster (14). The function of the major SREBP-1c isoform may be to support fatty acid biosynthesis, and the smaller amounts of SREBP-1a may support low rates of cholesterol biosynthesis as well. On a low cholesterol chow diet, the mouse liver produces relatively little SREBP-2. The situation changes when the liver is deprived of cholesterol by treatment with diets con-
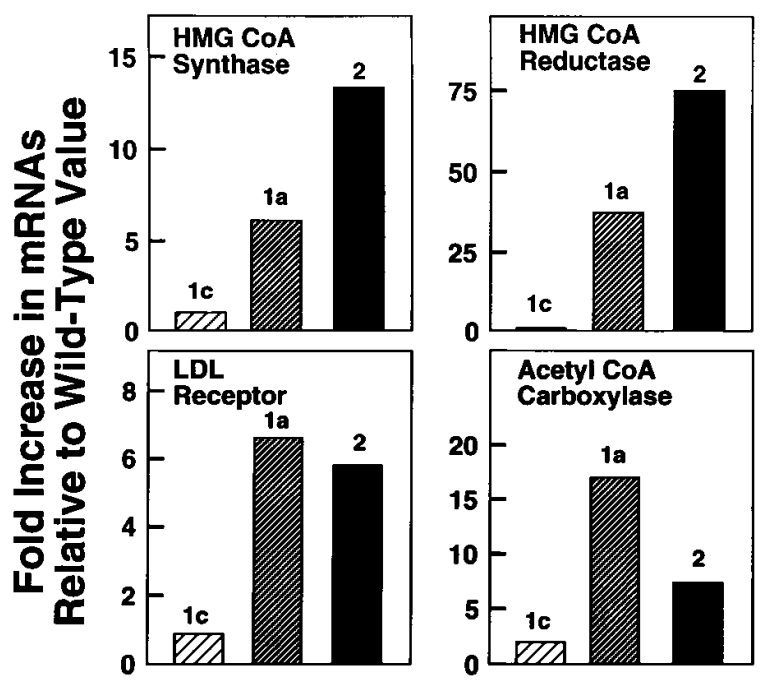

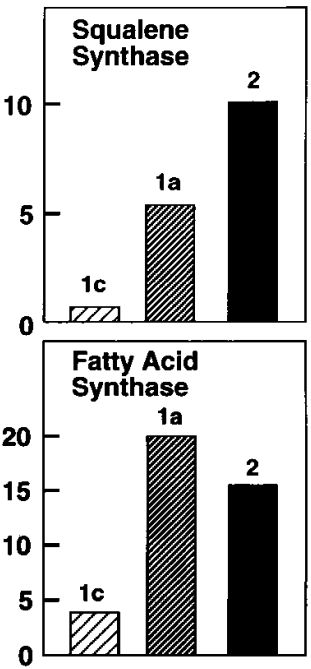

Figure 7. Relative levels of various mRNAs in livers of mice expressing transgenes encoding truncated SREBP-1c, -1a, and -2. Wild-type mRNA levels in each experiment are arbitrarily set at 1. The mRNA values for mice expressing truncated SREBP-1c (15) and SREBP-1a (16) were measured by Northern gel analysis and are taken from the indicated publication. mRNA levels for the transgenic SREBP-2 mice are from this paper. All mice were fed the low carbohydrate/high protein diet for $2 \mathrm{wk}$. Transgenic SREBP-1a and SREBP-2 mice were fasted for $4 \mathrm{~h}$ before killing. Transgenic SREBP-1c mice were not fasted. 

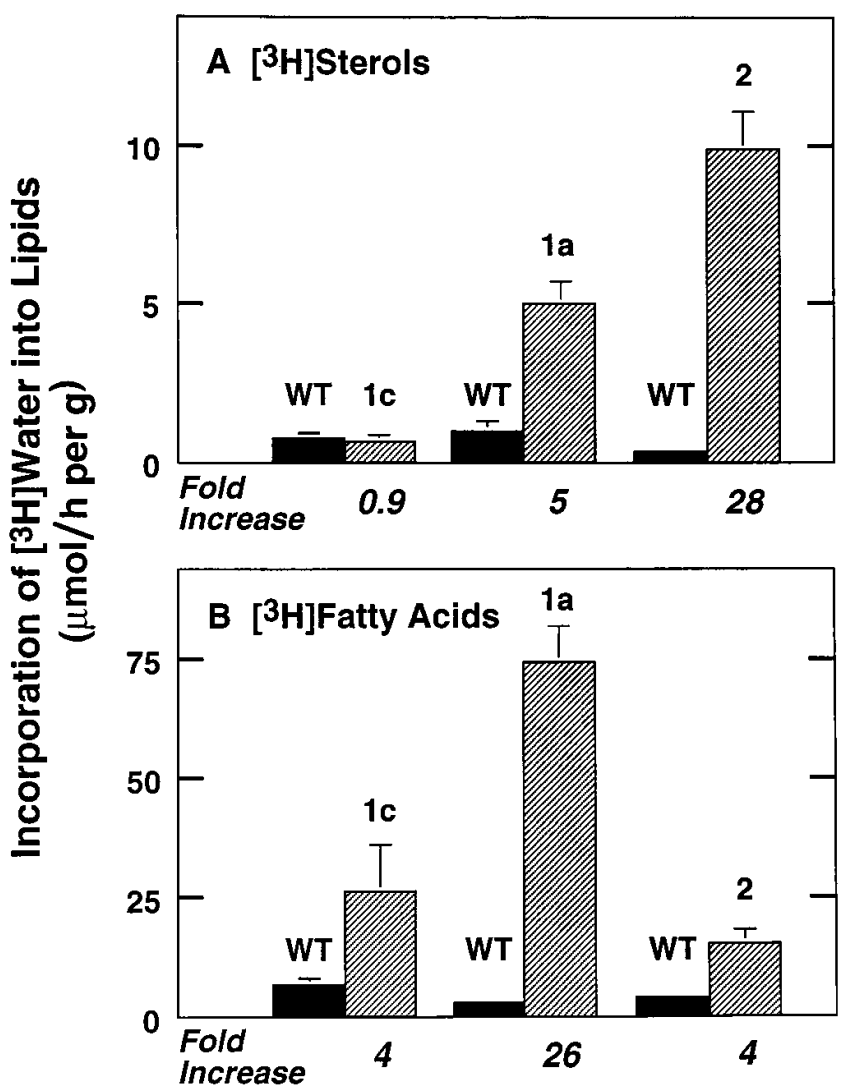

Figure 8. Rates of cholesterol and fatty acid synthesis in vivo in livers of mice expressing transgenes encoding truncated SREBP-1c, -1a, and -2. Values for SREBP-1a transgenic mice and their wild-type $(W T)$ controls (mean \pm SEM for 4-6 mice) are reproduced from experiments described in Table III in reference 16. Synthesis rates for SREBP-1c animals ( 7 mo of age) represent the mean \pm SEM of values from three female and one male transgenic SREBP-1c mice as compared with four sex-matched littermate wild-type mice. Synthesis rates for transgenic SREBP-2, line B mice (mean \pm SEM for five mice) are derived from the animals described in Table II. All mice were fed the low carbohydrate/high protein diet for $2 \mathrm{wk}$ before the experiment. The SREBP-1a and - 2 mice and their wild-type controls were fasted 2 and $4 \mathrm{~h}$, respectively, before killing. The SREBP-1c mice were studied in the nonfasted state. All mice were injected with $50 \mathrm{mCi}$ of $\left[{ }^{3} \mathrm{H}\right]$ water intraperitoneally, and $1 \mathrm{~h}$ later the liver was removed for measurement of its content of ${ }^{3} \mathrm{H}$-labeled digitonin-precipitable sterols and fatty acids as described in Methods.

taining an HMG CoA reductase inhibitor (lovastatin) and a bile acid-binding resin (Colestipol). Under these conditions, livers of hamsters (25) and mice (Shimano, H., J. Horton, J.L. Goldstein, and M.S. Brown, unpublished observations) downregulate the production of the SREBP-1 isoforms and they upregulate production of SREBP-2. The proteolytic cleavage of the SREBP-2 precursor is also enhanced, leading to a marked increase in the content of the $\mathrm{NH}_{2}$-terminal segment of SREBP-2 in liver nuclei (reference 25 and Shimano, H., J. Horton, J.L. Goldstein, and M.S. Brown, unpublished observations). The current data suggest that the increase in SREBP-2, at the expense of the SREBP-1 isoforms, would lead to a selective increase in cholesterol biosynthesis as opposed to fatty acid biosynthesis. This would be an appropriate response to cholesterol deficiency.

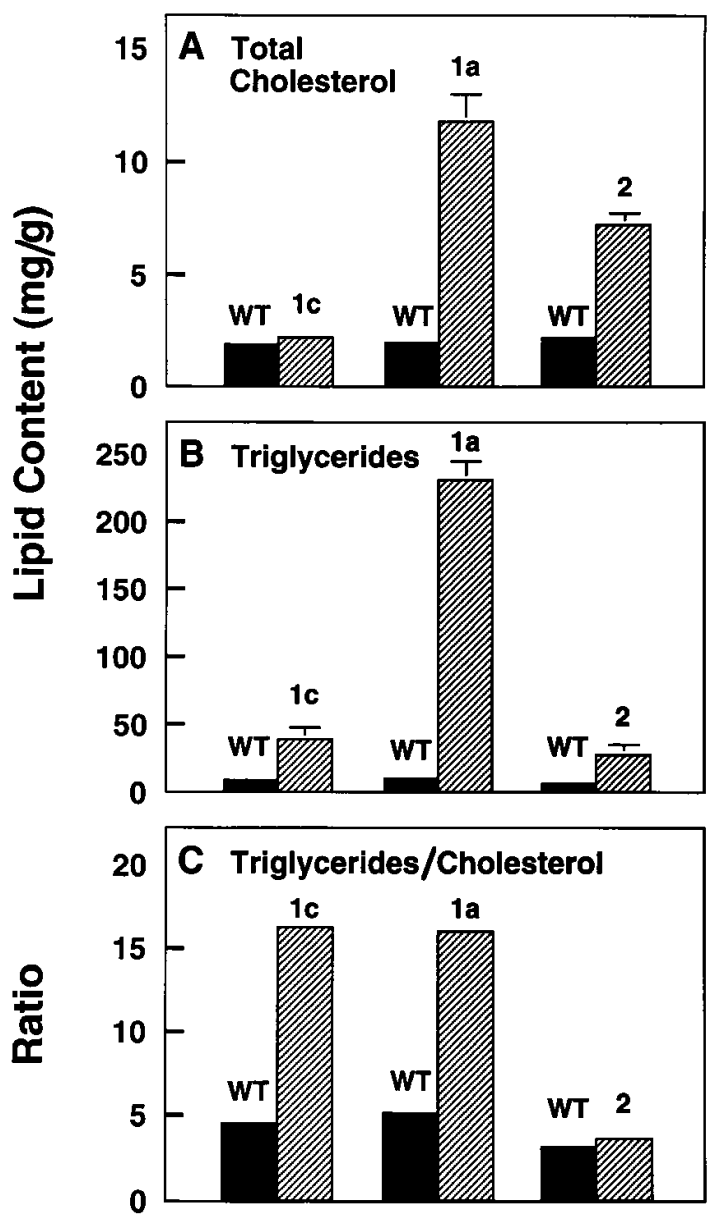

Figure 9. Comparison of cholesterol and triglyceride content in livers of mice expressing transgenes encoding truncated SREBP-1c, -1a, and -2. Values for the transgenic SREBP-1c mice and their wild-type $(W T)$ controls (mean \pm SEM for eight to nine mice) are taken from data of Table I in reference 15. Values for the transgenic SREBP-1a mice and their WT controls (mean \pm SEM for six mice) are taken from data of Table I, Exp. B in reference 16. Values for the transgenic SREBP-2, line B mice are from this paper. All mice were fed the low carbohydrate/high protein diet for $2 \mathrm{wk}$ before the experiment. Transgenic SREBP-1a and SREBP-2 mice were fasted for $4 \mathrm{~h}$ before killing. Transgenic SREBP-1c mice were not fasted.

The selective effect of SREBP-2 in stimulating cholesterol synthesis was particularly apparent in adipose tissue. Here the SREBP-2 transgenic mice showed a distinct increase in mRNAs encoding the LDL receptor and cholesterol biosynthetic enzymes yet no change in the mRNAs of the fatty acid pathway (Fig. 4). It seems likely that the potential stimulating effects of SREBP-2 on fatty acid-related genes is counterbalanced by other regulatory proteins that tend to limit fatty acid biosynthesis.

In addition to the differential effects of cholesterol and fatty acid biosynthesis, it is also possible that the three SREBP isoforms may have different effects on transcription of other genes whose existence is as yet unknown. Further elucidation of the effects of SREBPs on gene transcription will require detailed study of other genes that may be activated in the livers of the transgenic animals, as well as a more detailed comparison of the transcription-stimulating effects of the three iso- 
forms in tissue culture cells where a larger spectrum of quantitative measurements can be made. Studies along these lines are now underway.

\section{Acknowledgments}

We thank Yuri Bashmakov, Scott Clark, Shan Maika, and Robin Craddock for excellent technical assistance; Richard Gibson and Laura Quinlivan for invaluable help with the animals; and Dr. Laura Woollett for assistance with the $\left[{ }^{3} \mathrm{H}\right]$ water experiments.

This work was supported by grants from the National Institutes of Health (HL-20948), the Moss Heart Foundation, and the Perot Family Foundation. J.D. Horton is the recipient of a Postdoctoral Fellowship for Physicians from the Howard Hughes Medical Institute (Dallas, TX). I. Shimomura is the recipient of a Research Fellowship from the Manpei Suzuki Diabetes Foundation of Tokyo, Japan.

\section{References}

1. Dietschy, J.M. 1997. Overview of cholesterol and lipoprotein metabolism in the brain, liver and extrahepatic organs. Nutr. Metab. Cardiovasc. Dis. 7:162-168.

2. Hillgartner, F.B., L.M. Salati, and A.G. Goodridge. 1995. Physiological and molecular mechanisms involved in nutritional regulation of fatty acid synthesis. Physiol. Rev. 75:47-76.

3. Brown, M.S., and J.L. Goldstein. 1997. The SREBP pathway: Regulation of cholesterol metabolism by proteolysis of a membrane-bound transcription factor. Cell. 89:331-340.

4. Kim, J.B., G.D. Spotts, Y.-D. Halvorsen, H.-M. Shih, T. Ellenberger, H.C. Towle, and B.M. Spiegelman. 1995. Dual DNA binding specificity of ADD1/SREBP1 controlled by a single amino acid in the basic helix-loop-helix domain. Mol. Cell. Biol. 15:2582-2588.

5. Yokoyama, C., X. Wang, M.R. Briggs, A. Admon, J. Wu, X. Hua, J.L. Goldstein, and M.S. Brown. 1993. SREBP-1, a basic helix-loop-helix leucine zipper protein that controls transcription of the LDL receptor gene. Cell. 75 : 187-197.

6. Hua, X., C. Yokoyama, J. Wu, M.R. Briggs, M.S. Brown, J.L. Goldstein, and X. Wang. 1993. SREBP-2, a second basic-helix-loop-helix-leucine zipper protein that stimulates transcription by binding to a sterol regulatory element. Proc. Natl. Acad. Sci. USA. 90:11603-11607.

7. Ericsson, J., S.M. Jackson, B.C. Lee, and P.A. Edwards. 1996. Sterol regulatory element binding protein binds to a cis element in the promoter of the farnesyl diphosphate synthase gene. Proc. Natl. Acad. Sci. USA. 93:945-950.

8. Guan, G., P.-H. Dai, T.F. Osborne, J.B. Kim, and I. Shechter. 1997. Multiple sequence elements are involved in the transcriptional regulation of the human squalene synthase gene. J. Biol. Chem. 272:10295-10302.

9. Kim, J.B., and B.M. Spiegelman. 1996. ADD1/SREBP1 promotes adipocyte differentiation and gene expression linked to fatty acid metabolism. Genes \& Dev. 10:1096-1107.

10. Lopez, J.M., M.K. Bennett, H.B. Sanchez, J.M. Rosenfeld, and T.F. Osborne. 1996. Sterol regulation of acetyl CoA carboxylase: A mechanism for co- ordinate control of cellular lipid. Proc. Natl. Acad. Sci. USA. 93:1049-1053.

11. Ericsson, J., S.M. Jackson, J.B. Kim, B.M. Spiegelman, and P.A. Edwards. 1997. Identification of glycerol-3-phosphate acyltransferase as an adipocyte determination and differentiation factor 1 and sterol regulatory elementbinding protein-responsive gene. J. Biol. Chem. 272:7298-7305.

12. Shimomura, I., H. Shimano, J.D. Horton, J.L. Goldstein, and M.S Brown. 1997. Differential expression of exons 1a and 1c in mRNAs for sterol regulatory element binding protein-1 in human and mouse organs and cultured cells. J. Clin. Invest. 99:838-845.

13. Tontonoz, P., J.B. Kim, R.A. Graves, and B.M. Spiegelman. 1993. ADD1: a novel helix-loop-helix transcription factor associated with adipocyte determination and differentiation. Mol. Cell. Biol. 13:4753-4759.

14. Shimomura, I., Y. Bashmakov, H. Shimano, J.D. Horton, J.L. Goldstein, and M.S. Brown. 1997. Cholesterol feeding reduces nuclear forms of sterol regulatory element binding proteins in hamster liver. Proc. Natl. Acad. Sci. USA. 94:12354-12359.

15. Shimano, H., J.D. Horton, I. Shimomura, R.E. Hammer, M.S. Brown, and J.L. Goldstein. 1997. Isoform 1c of sterol regulatory element binding protein is less active than isoform 1a in livers of transgenic mice and in cultured cells. J. Clin. Invest. 99:846-854.

16. Shimano, H., J.D. Horton, R.E. Hammer, I. Shimomura, M.S. Brown, and J.L. Goldstein. 1996. Overproduction of cholesterol and fatty acids causes massive liver enlargement in transgenic mice expressing truncated SREBP-1a J. Clin. Invest. 98:1575-1584.

17. Hamilton, J.G., and K. Comai. 1988. Rapid separation of neutral lipids, free fatty acids and polar lipids using prepacked silica Sep-pak columns. Lipids. 23:1146-1149.

18. Turley, S.D., M.W. Herndon, and J.M. Dietschy. 1994. Reevaluation and application of the dual-isotope plasma ratio method for the measurement of intestinal cholesterol absorption in the hamster. J. Lipid Res. 35:328-339.

19. Short, M.K., D.E. Clouthier, I.M. Schaefer, R.E. Hammer, M.A. Magnuson, and E.G. Beale. 1992. Tissue-specific, developmental, hormonal, and dietary regulation of rat phosphoenolpyruvate carboxykinase-human growth hormone fusion genes in transgenic mice. Mol. Cell. Biol. 12:1007-1020.

20. Chomczynski, P., and N. Sacchi. 1987. Single-step method of RNA isolation by acid guanidinium thiocyanate-phenol-chloroform extraction. Anal. Biochem. 162:156-159.

21. Shimano, H., I. Shimomura, R.E. Hammer, J. Herz, J.L. Goldstein, M.S. Brown, and J.D. Horton. 1997. Elevated levels of SREBP-2 and cholesterol synthesis in livers of mice homozygous for a targeted disruption of the SREBP-1 gene. J. Clin. Invest. 100:2115-2124.

22. Zhu, Y., K. Alvares, Q. Huang, M.S. Rao, and J.K. Reddy. 1993. Cloning of a new member of the peroxisome proliferator activated receptor gene family from mouse liver. J. Biol. Chem. 268:26817-26820.

23. Sato, R., J. Yang, X. Wang, M.J. Evans, Y.K. Ho, J.L. Goldstein, and M.S. Brown. 1994. Assignment of the membrane attachment, DNA binding, and transcriptional activation domains of sterol regulatory element binding protein-1 (SREBP-1). J. Biol. Chem. 269:17267-17273.

24. Sharp, D., L. Blinderman, K.A. Combs, B. Kienzle, B. Ricci, K. WagerSmith, C.M. Gil, C.W. Turck, M.-E. Bouma, D.J. Rader, et al. 1993. Cloning and gene defects in microsomal triglyceride transfer protein associated with abetalipoproteinaemia. Nature. 365:65-69.

25. Sheng, Z., H. Otani, M.S. Brown, and J.L. Goldstein. 1995. Independent regulation of sterol regulatory element binding proteins 1 and 2 in hamster liver. Proc. Natl. Acad. Sci. USA. 92:935-938. 